\title{
Experiencia del CEIMM en Las Minas: Acompaña- miento a las mujeres en los territorios indígenas ${ }^{1}$
}

\author{
CEIMM in Las Minas Experience: Accompanying women in indigenous territories
}

\author{
Jacoba del Carmen Dávila Molina²
}

\section{Resumen}

Este artículo describe cómo se desarrolló el proceso de acompañamiento en los territorios indígenas, fundamentalmente el trabajo con las mujeres, las principales dificultades, alcances y desafíos. Acompañamiento comunitario que la Universidad de las Regiones Autónomas de la Costa Caribe Nicaragüense (URACCAN), a través del Centro de Estudio e Información de la Mujer Multiétnica (CEIMM), ha brindado en la búsqueda de promover cambios de actitudes con relación a la práctica de relaciones equitativas de género, fortalecimiento de la organización, el liderazgo, autonomía de las mujeres y los pueblos, esto accede a la convivencia comunitaria, la conservación de los recursos naturales, promoción de la incidencia en las políticas públicas y el diálogo intercultural. Así mismo, facilitar la comunicación y confianza comunitaria, con autoridades municipales, regionales o nacionales para el empoderamiento de los pueblos y de las mujeres. Una de las situaciones que preocupa es la poca participación activa de las mujeres en los diferentes procesos de desarrollo de las comunidades indígenas ya que, por historia, no existe un reconocimiento de sus capacidades.

Palabras clave: Empoderamiento; convivencia armónica; autonomía; diálogo intercultural; incidencia; autogestión.

\section{Abstract}

This article describes how the accompaniment process was developed in the indigenous territories, mainly the work with women, the main difficulties, achievements and challenges. The Community accompaniment supported by the University of the Autonomous Regions of the Nicaraguan Caribbean Coast (URACCAN) through the Center for the Study and Information of Multiethnic Women (CEIMM). It has offered a promotion of attitude changes, in relation to the practice of gender equitable relationships, organization strengthening, leadership, women and peoples autonomy to create access to community coexistence, natural resources conservation, promotion of advocacy in public policies and intercultural dialogue. Likewise, to facilitate community communication and trust, with municipal, regional or national authorities for the empowerment of peoples and women. One of the concerns is the low level of active women participation in the different development processes of indigenous communities since, by history, there is no recognition of their capabilities.

Key words: Empowerment; harmonious coexistence; autonomy; intercultural dialogue: incidence; self-management.

\footnotetext{
1 XIV Congreso Latinoamericano y del Caribe de Extensión Universitaria y Segundo Congreso Centroamericano de Compromiso Social para la vinculación socia de la universidad con la sociedad.

2 Doctora en Educación con especialidad en mediación pedagógica. Coordinadora del Centro de Estudio e Información de la Mujer Multiétnica de la Universidad de las Regiones Autónomas de la Costa Caribe Nicaragüense - Recinto Universitario Las Minas. Correo: jacobamolina2013@gmail.com ORCID: https://orcid.org/0000-0001-8831-0019
}

Recibido: 17/01/2018 - Aprobado: 05/04/2018 
Historia y actualidad de URACCAN

\section{Introducción}

La URACCAN con su rol de institución académica formadora, visibilizada en su misión, visión así como en los ejes transversales; desde su fundación contribuye al fortalecimiento de los procesos de autogestión y autodesarrollo de los pueblos indígenas, afrodescendientes y mestizos, a través de programas, acciones y tareas que conllevan a mejorar las condiciones de vida de mujeres y hombres, así mismo en la creación de espacios de participación, debate y reconocimiento de los derechos de las mujeres tanto a nivel interno de la universidad como externo, desde el marco de los derechos autonómicos.

Su modelo académico de extensión social y comunitaria se crea a partir de las demandas de la sociedad de la Costa Caribe de Nicaragua, para formar localmente los recursos humanos, a través del fortalecimiento de modelos locales en los distintos campos de la sociedad Costeña.

En este sentido, su acompañamiento resalta en mujeres indígenas quienes históricamente no han ejercido sus derechos sociales, económicos, políticos, culturales, sexuales y reproductivos. Su lucha por ser reconocidas como sujetas de derechos se remonta a la época de la independencia, pero el surgimiento y la existencia del movimiento de mujeres como tal, es más reciente y se ubica a inicios del siglo XX. Este proceso de acompañamiento para las mujeres indígenas es relevante, porque contribuye al ejercicio efectivo de sus derechos.

En la Costa Caribe nicaragüense tanto en el norte como en el sur, incluyendo el Triángulo Minero, las organizaciones de mujeres desarrollan un papel fundamental en cuanto al fomento de capacidades, reconocimiento y ejerciendo sus derechos como mujeres multiétnicas. Se destacan las organizaciones de mujeres indígenas quienes con sus luchas han generado cambios en los pensamientos de la membrecía de sus organizaciones y ha trascendido hacia actores públicos para convertirlos en aliados estratégicos en la búsqueda de una igualdad real en el respeto y acceso a la justicia de las mujeres indígenas.

Pese a todo el esfuerzo que las mujeres han realizado a través del acompañamiento de organizaciones como URACCAN, aún nos queda un largo camino por recorrer para alcanzar un mundo mejor, con justicia social y conseguir la convivencia armónica.

\section{Revisión de literatura}

Los movimientos de mujeres en la región centroamericana han logrado incidir en sus Estados y gobiernos, de manera que a 20 años de la Conferencia Internacional sobre la población y el desarrollo de El Cairo, llevada a cabo en Egipto en 1994, existen leyes y políticas públicas nacionales a favor de la igualdad entre mujeres y hombres. En Guatemala desde el 2001 se aprobó su Política de promoción y desarrollo de las mujeres y su Plan de equidad de oportunidades, en Honduras se aprobó desde el año 2000 y en Nicaragua se aprobó hasta el año 2008. Sólo en Nicaragua existe la paridad en los cargos de elección por ley y ha sido voluntad política del gobierno desde el 2007 la ocupación de cargos de toma de decisión, ministerios y vice ministerios (Voces Caribeñas O. d., 2014).

La relevancia de la participación comunitaria y principalmente de las mujeres en los procesos de organización, planificación y gestión territorial, en el actual debate acerca del desarrollo local, queda confirmada por el gran número de gestiones asociadas con el tema desarrollo integral comunitario. Los argumentos teórico-conceptuales, relativos a la planificación comunitaria y la participación en los procesos de decisión, tocan diferentes aspectos entre los cuales destacan el desarrollo comunitario sostenible, la gestión de los recursos por parte de las comunidades, con base en el principio de la equidad ecológica, y las prácticas de participación ciudadana (Fracasso, 2000).

La Constitución Política de Nicaragua reconoce el derecho de toda la población a organizarse. En específico el Capítulo II de los Derechos Políticos, en el artículo 49. Refiere que en Nicaragua tienen derecho a constituir organizaciones trabajadores y trabajadoras del Campo y la ciudad, mujeres, jóvenes, producto- 
res, agropecuarios, artesanos, profesionales, técnicos, intelectuales, artistas, religiosos, las comunidades de la Costa Caribe y población en general, sin discriminación alguna, con el fin de lograr la realización de sus aspiraciones según sus propios intereses y participar en la construcción de una nueva sociedad.

En el capítulo II: Comunidades de la Costa Caribe: Artículo 18o. Dice que las comunidades de la Costa Caribe tienen el derecho inalienable de vivir y desarrollarse bajo la forma de organización político-administrativa, social y cultural que corresponde a sus tradiciones históricas y culturales. Sin embargo, aun contando con este marco legal, las organizaciones civiles en específico las organizaciones de mujeres que trabajan en su mayoría por reivindicar sus derechos, son las que tienen menos oportunidades para poder mantenerse en el tiempo por las políticas implementadas por el Estado.

Nicaragua cuenta con una Ley de Participación Ciudadana que crea, un sistema que va desde lo nacional a lo local. Por otro lado, la Ley establece la participación de la ciudadanía en la formulación de políticas públicas a todo nivel através de un sistema de consejos de participación.

Un aspecto novedoso de esta Ley es la posibilidad que abre a la población a nivel municipal de organizar las Asociaciones de Pobladores. Esta es una figura asociativa interesante. Aparece esbozada en el artículo 37 de la Ley de Municipios: "el Concejal Municipal apoyará la creación de asociaciones de pobladores que tengan como fin el desarrollo municipal y fomentará la participación de las organizaciones y asociaciones sectoriales, culturales, gremiales, deportivas, profesionales y otras en la gestión municipal". La Ley de Ordenamiento y Titulación de Asentamientos Humanos Espontáneos (Ley 309) da un rol en el proceso de estudio sobre la posible legalización de un asentamiento. La Ley de Participación Ciudadana señala formas de constitución, requisitos básicos para su reconocimiento en el municipio, su funcionamiento y las finalidades para las que puede conformarse una asociación de pobladores.

Las reivindicaciones del movimiento indígena han partido en muchos casos de problemas concretos que de modo recurrente aparecen en la mayoría de los documentos de las organizaciones indígenas, pero a medida que el movimiento se fue articulando mejor las reivindicaciones concretas han perdido su carácter aislado y localista y se han ido situando cada vez más en el marco de la reivindicación de los derechos de los pueblos indígenas (UNESCO, 2008).

La gobernanza territorial es reconocida en la Declaración de las Naciones Unidas sobre los derechos de los pueblos indígenas establecido en el Convenio 169 de la OIT (organización internacional de trabajadores), en la Constitución Política de Nicaragua de 1987 y en el Estatuto de Autonomía de las Regiones Autónomas de la Costa Caribe de Nicaragua en 1987 y la Ley 445, Régimen de Propiedad Comunal de los Pueblos Indígenas de las Regiones Autónomas de la Costa Caribe de Nicaragua y de los ríos Bocay, Coco, Indio y Maíz en 2003, que las autoridades indígenas contaron con una base legal operativa para recuperar sus territorios históricos a través de un reconocimiento público de su patrimonio y de su propiedad colectiva.

La organización comunitaria y territorial en los pueblos indígenas, estuvo orientada a brindar servicios en gestión social comunitaria que respondieran a los intereses económicos, políticos, sociales y culturales de los grupos hegemónicos del Estado y no a las necesidades y expectativas de las comunidades indígenas. Las políticas de gestión y planificación comunitaria, los planes y programas sociales fueron siempre diseñados desde el gobierno central y municipal con pensamientos diferentes, donde no existe una explícita claridad de la perspectiva de género desde el conocimiento endógeno y exógeno (UNESCO, 2008).

Gargallo (2014), expresa:

Las mujeres somos la mitad de todos los pueblos. Y en todos los pueblos, hemos generado un pensamiento crítico a la organización desigual de los poderes entre hombres y mujeres, una teorización liberal sobre la abstracta igualdad de las mujeres y los hombres o la búsqueda concreta emprendida por las mujeres para el bienestar de las mismas y en diálogo entre sí para destejer los 
símbolos y prácticas sociales que las ubican en un lugar secundario, con menos derechos y una valoración menor que los hombres. (p.10)

Existen iniciativas valiosas como la propuesta de reformas a la Ley 28. Estatuto de Autonomía de las Regiones Autónomas de la Costa Caribe de Nicaragua, que ante la ausencia de enfoque de género y multicultural, presentó Voces Caribeñas en el 2008, en el proceso encaminado a la definición de una agenda de las mujeres costeñas, la propuesta fue presentada al Gobierno Regional para que fuese canalizada ante la Asamblea legislativa, pero seis años después la propuesta no ha prosperado (Asociación Red de Mujeres Afrodescendientes, 2014).

Según Faúndez y Weinstein (2011), "la incorporación de la dimensión de género en las políticas públicas, ha tenido en su breve desarrollo, expresiones diversas. Cada una de estas propuestas muestra diferentes alternativas de encarar este desafío" (p. 53). Según estas autoras, las mujeres desde sus orígenes han manifestado la necesidad de identificar, reconocer y visibilizar sus derechos como grupo específico:

En el contexto de las estrategias anti pobreza de los años 80'y 9o', las políticas dirigidas a las mujeres se desarrollaron situándolas casi única y exclusivamente en su calidad de madres y comenzaron a priorizarlas como un grupo de riesgo o vulnerable, las cuales, junto a los niños, los discapacitados y los ancianos, fueron consideradas grupos socialmente débiles o vulnerables desprovistos de toda la capacidad para decidir y aportar al desarrollo (p. 66).

Este enfoque, que retomó criterios asociados con las antiguas estrategias del bienestar social, "tuvo como expresión durante décadas, políticas y programas que consideraban a las mujeres bajo determinados supuestos, muchas veces ocultos en las agendas de las políticas públicas” (p. 70).

Faúndez y Weinstein (2011), refieren que el enfoque de género es útil y necesario en diferentes áreas de la vida social, tanto para el mundo público como privado:

Desde las políticas económicas y sociales a nivel macro, los planes de desarrollo estratégicos sectoriales, hasta programas y proyectos locales en comunidades y contextos muy específicos. La superación de la desigualdad entre mujeres y hombres, como la de cualquier otra desigualdad, es un requisito para avanzar al logro de objetivos amplios de desarrollo, al permitir que cada cual desarrolle su potencial humano con integridad y dignidad independientemente de su sexo (p. 87).

\section{Materiales y método}

El proceso de acompañamiento a las mujeres indígenas mayangnas, se realizó en tres territorios: Sauni Arunka, Sauni As y Sauni Bas, ubicados en los municipios de Siuna y Bonanza en la Costa Caribe Norte durante 2010 - 2016. El trabajo consistió en la atención directa, a través de visitas a los territorios indígenas donde la universidad brinda acompañamiento, grupos de reflexión, intercambios de experiencias, capacitaciones y procesos formativos. La población involucrada en el proceso fueron mujeres, líderes comunales y territoriales. La práctica de la extensión social comunitaria se hizo con el consentimiento previo, lo que significó establecer un proceso de comunicación equitativa, horizontal, participativa con las comunidades para el desarrollo del acompañamiento según las demandas de las mismas. 


\section{Resultados y discusión}

El proceso de acompañamiento que la URACCAN realiza en los territorios, se fundamenta en la aplicación de metodologías colaborativas y participativas que promueven el cambio de actitudes, el empoderamiento, la auto gestión comunitaria, la transformación local por el bienestar colectivo con perspectiva a largo plazo, basadas en criterios de sostenibilidad, el respeto a la cosmovisión y la convivencia.

\section{Extensión social y comunitaria}

Este modelo de extensión social y comunitaria nace como respuesta a demanda de la sociedad costeña nicaragüense para formar localmente los recursos humanos, a través del acompañamiento de espacios locales en distintos aspectos: sociales, políticos, ambientales y económicos que contribuyan a fortalecer los procesos autonómicos.

Por lo que la extensión social y comunitaria se define como los procesos y acciones que realiza la URACCAN en y con las comunidades y su liderazgo, la comunidad en su conjunto, autoridades comunitarias, municipales y regionales, así con organizaciones sociales y productivas, con el fin de un mejor aprovechamiento de los recursos y sus capacidades para la convivencia en armonía.

\section{Características del acompañamiento}

El acompañamiento está centrado en las capacidades de las personas y en el desarrollo de sus potencialidades, responde a un proceso de desarrollo social, ecológico y económico sostenible y no al cumplimiento de las metas de un proyecto, se enmarca más en los cambios de actitudes, que en la forma de realizar las actividades, se realiza en un ambiente natural y se apega a las condiciones comunitarias, motiva a las personas a resolver problemas, trabajando con ellas y ellos; promueve acciones críticas, sin intervenir en las tomas de decisiones que las comunidades definen.

\section{Acciones desarrolladas en el proceso de acompañamiento}

Este proceso se realizó en tres fases: La primera fase se desarrolló del 2010 al 2012, esta consistió en un acercamiento directo con el territorio, es decir hacer empatía con líderes y lideresas de los tres territorios, conocer sus necesidades, aquí se realizó un diagnóstico comunitario para conocer la realidad y demandas de las mujeres, donde a partir de ello se hizo el proceso de acompañamiento a través de las siguientes estrategias: 1. Grupos de concertación, 2.Grupos de reflexión y 3. Diplomado.

Grupos de concertación: Estos grupos de concertación consistieron en diálogos abiertos entre líderes, lideresas comunales, territoriales y universidad, para realizar acciones de manera coordinada y desarrollar los procesos de acompañamiento a las mujeres en los territorios, con el consentimiento de la comunidad.

Grupos de reflexión: En estos grupos participaron mujeres de los diferentes territorios seleccionadas por sus representantes, consistió en reflexionar sobre temáticas de género, autoestima, derechos humanos, violencia, entre otros.

Diplomado: Se implementó un diplomado sobre derechos humanos con énfasis en los derechos de las mujeres, conformado por mujeres con algún tipo de participación en el territorio y lideresas, este proceso se realizó de manera rotativa en dos comunidades céntricas a los territorios.

Segunda fase 2013 al 2014. Se dio la construcción de un tejido social comunitario con enfoque de territorio desde la perspectiva intercultural de género. Aquí se crearon organizaciones de mujeres a nivel local, territorial y nacional; desarrollo de intercambios de experiencias entre organizaciones tanto a nivel departamental como a nivel nacional. 
Tercera fase 2015 al 2017. Consistió en la consolidación de las organizaciones de mujeres, donde realizaron sus agendas de desarrollo, planificación estratégica y su proyecto de vida.

\section{Alcances del proceso de acompañamiento}

1. El fortalecimiento de capacidades para la autogestión comunitaria y tomas de decisiones, como parte de la restitución del poder, sobre la base de los derechos de las mujeres.

2. La pertinencia cultural, se fundamenta en la comprensión y aplicación de conceptos vinculados al desarrollo como la interculturalidad, género, desarrollo sostenible conocimiento tradicional, el diálogo intercultural y el relevo generacional

3. Romper el hielo y escuchar sus voces, sin miedo ni timidez, enfrentándose a espacios públicos tanto a nivel local, regional como nacional e internacional.

4. Conformación de organizaciones de mujeres microempresarias, cooperativas de producción en cacao y artesanía a nivel comunal, territorial y la organización de gobierno de mujeres mayangna a nivel nacional.

5. Algunas organizaciones de mujeres cuentan con infraestructura propia para su funcionamiento, con personería jurídica, estatus y reglamentos internos

\section{Debilidades}

1. Poca coordinación entre la membresía de la organización, desinterés de parte de algunas socias en realizar trabajo voluntario cuando no hay apoyo económico para las actividades, no hay articulación entre líderes comunales, territorial y nación mayangna.

2. No existe trabajo coordinado con otras organizaciones comunales para fortalecer el accionar de las organizaciones, poco o nulo apoyo de las organizaciones gubernamentales, debilidad en el uso y manejo de los fondos.

\section{Desafía o retos:}

1. Avanzar hacia el desarrollo con identidad y crear una cultura empresarial, fortalecimiento de las organizaciones de mujeres, facilitar la presencia de mujeres en los espacios de participación y el relevo generacional.

2. Establecer diálogos y reflexiones con líderes, lideresas sobre los derechos humanos de las personas.

3. Trabajar el plan de vida del gobierno de las mujeres, finalizar con el documento de estrategia de visibilización de las mujeres

4. Mayor acceso a la educación formal, continuar con el proceso de capacitación en el tema de género y derechos humanos, promoción y defensa de la identidad cultural.

5. Gestionar para una mayor cobertura en la atención a mujeres, ubicando puestos de salud en lugares de difícil acceso, una atención en salud desde el enfoque intercultural donde se promueva el uso de la medicina tradicional para el tratamiento de algunas enfermedades y alternándola con la medicina occidental en caso necesario.

6. Promover alternativas económicas para el desarrollo de los colectivos de artesanías que permita contar con recursos necesarios y garantizar la calidad de los productos. 
7. Desarrollar programas culturales en lengua materna que aborden sobre las diferentes culturas, los elementos de valor cultural, la filología (la escritura, la gramática), divulgaciones de las leyes tales como la ley 779.

\section{Conclusiones}

El trabajo de acompañamiento a los territorios indígenas, dio paso a la creación de espacios de concertación y debate con diferente actores claves tanto a nivel local como territorial; sin embargo, hace falta continuar fortaleciendo estos espacios donde se armonice un ambiente de respeto a los saberes y el diálogo entre mujeres y hombres de los territorios indígenas.

Nuestros retos son avanzar hacia el desarrollo con identidad, crear una cultura empresarial, fortalecer las organizaciones de mujeres, facilitar la presencia de comunitarios y comunitarias en los espacios de participación y el relevo generacional.

Las condiciones económicas actuales de las organizaciones de mujeres mayangnas en este momento no son las mejores, pues cada día es más difícil conseguir apoyo de la cooperación externa; para subsistir han creado iniciativas que les permita la generación de ingresos para el funcionamiento y sostenibilidad de sus organizaciones.

\section{Lista de referencias}

Comisión Económica para América Latina y el Caribe (CEPAl,2013). Estudio Económico de América Latina y el Caribe 2013: tres décadas de crecimiento económico desigual e inestable.

Faúndez Alejandra \& Weinstein Marisa (2011). Para incorporar enfoque de género en la gestión pública con Población Indígena Ciudad de México.

Fracasso L. (2000). PLANIFICACIÓN COMUNITARIA Y PARTICIPACIÓN EN LOS PROCESOS DE DECISIÓN: CATEGORÍAS DE ANÁLISIS Y ARGUMENTOS. Revista Bibliográfica de Geografía y Ciencias Sociales Universidad de Barcelona [ISSN 1138-9796] $\mathrm{N}^{\circ} 216,8$ de marzo de 2000

Gargallo. Celentani F. (2014). Feminismos desde Abya Yala. Ideas y proposiciones de las mujeres de 607 pueblos en nuestra AMÉRICA. Editorial Corte y Confección, Ciudad de México, Primera edición digital.

Organización de las Naciones Unidas para la Educación, la Ciencia y la Cultura (Unesco, 2008). La educación inclusiva: el camino hacia el futuro.

Valiente, Fredy y Cid, Víctor (2007). Sistematización del proceso de creación y desarrollo de la Universidad URACCAN en el Triángulo Minero Período 1995 - 2005.

URACCAN. Informes técnicos de los procesos de acompañamiento de 2007 al 2015.

Voces Caribeñas, O. d. (2014). Agenda de las mujeres de la costa Caribe Nicaraguense. Agenda de mujeres de la Costa Caribe Nicaraguense, Bilwi, Puerto Cabezas, RAAN. Recuperado el Julio de 2014. 